\title{
Charles Sorel, Les nouvelles choisies. Où se trouvent divers incidents d'Amour et de Fortune
}

\section{Filippo D'angelo}

\section{(2) OpenEdition}

1 Journals

\section{Edizione digitale}

URL: http://journals.openedition.org/studifrancesi/27096

DOI: 10.4000/studifrancesi.27096

ISSN: 2421-5856

\section{Editore}

Rosenberg \& Sellier

\section{Edizione cartacea}

Data di pubblicazione: 31 décembre 2006

Paginazione: 561-563

ISSN: 0039-2944

\section{Notizia bibliografica digitale}

Filippo D'angelo, «Charles Sorel, Les nouvelles choisies. Où se trouvent divers incidents d'Amour et de Fortune », Studi Francesi [Online], 150 (L | III) | 2006, online dal 30 novembre 2015, consultato il 08 novembre 2020. URL : http://journals.openedition.org/studifrancesi/27096 ; DOI : https://doi.org/ 10.4000/studifrancesi.27096

\section{(c) (i) $\odot$}

Studi Francesi è distribuita con Licenza Creative Commons Attribuzione - Non commerciale - Non opere derivate 4.0 Internazionale. 
CHARLES SOREL, Les nouvelles choisies. Où se trouvent divers incidents d'Amour et de Fortune, édition critique par Daniela Dalla Valle, Paris, Honoré Champion, coll. «Sources classiques», 2005, pp. 429.

Il volume delle Nouvelles choisies curato da Daniela Dalla Valle inaugura per i tipi di Honoré Champion un ciclo di riedizioni di alcune fra le maggiori opere di Charles Sorel. Secondo un disegno editoriale teso a rinnovare la ricezione del grande poligrafo francese, al restauro filologico e al commento critico delle Nouvelles prodotti da Daniela Dalla Valle dovrebbero seguire, per mano di altri studiosi, analoghe operazioni sui testi dell'Histoire comique de Francion, del Berger extravagant e della Bibliothèque française. Tale progetto si giustifica per il peso crescente di Sorel negli studi sul Seicento francese (basti pensare all'inclusione del Francion fra le opere in programma per il Concours d'Agrégation del 2001, accompagnata dall'abituale, imponente massa bibliografica), ma trova ulteriore motivazione nelle peculiarità stesse della prassi letteraria dell'autore, costantemente impegnato in lavori di correzione, incremento e riscrittura.

Les Nouvelles choisies rappresentano in questa prospettiva un episodio centrale, vera e propria chiave di accesso ai sinuosi percorsi creativi di Sorel, e caso tanto più interessante in quanto, sino ad ora, poco indagato, se non altro rispetto alle numerose analisi di cui è stata oggetto la decennale stesura e revisione del Francion (si segnalano, in particolare modo, gli interventi di Jean Serroy, D'un roman à métamorphose: la composition du "Francion" de Charles Sorel, «Baroque», 6, 1973, pp. 97-103; Fausta Garavini, "Francion” rivisitato: diacronia di una struttura, «Saggi e ricerche di letteratura francese», XIV, 1975, pp. 39-107; e Emmanuel Desiles, Les avatars de l'Histoire comique de Francion, «Studi francesi», 120, XL, 1996, pp. 501-516). Come rimarcato da Daniela Dalla Valle a pagina 10 dell' introduzione, il legame genetico tra le due opere si presta d'altronde a un esame storico unitario. Entrambe vengono pubblicate, nella loro prima veste, lo stesso anno del processo contro Théophile de Viau, il 1623, data cruciale per la crisi della corrente libertina nella quale naviga l'allora giovanissimo Sorel. Entrambe, inoltre, verranno successivamente sottoposte a considerevoli aggiunte e rimaneggiamenti, questi ultimi spesso ispirati proprio dalla necessità di smorzare il libertinismo flamboyant delle versioni originali. Per quanto concerne Les Nouvelles choisies, il passaggio dalla prima edizione, il cui titolo era Les Nouvelles françaises, a quella del 1645, assunta come testo di riferimento dalla curatrice, rivela sfaccettature ideologiche ed estetiche non meno rilevanti di quelle già messe in luce dalla critica a proposito delle varianti del Francion.

Nell'esauriente introduzione al volume, Daniela Dalla Valle suddivide in cinque categorie le modifiche intervenute fra il 1623 e il 1645. Alcune di esse riguardano le cinque novelle già presenti nell'edizione originale, e consistono: la prima, in un'operazione di modernizzazione linguistica; la seconda, nella trasformazione dei titoli e nella mutata disposizione dei testi all'interno della raccolta; la terza, infine, nella già accennata attenuazione dei motivi più audacemente libertini. Le due ultime categorie vertono invece sugli innesti dell' edizione del 1645: innanzitutto, l'aggiunta di due testi inediti, rispettivamente all'inizio e alla fine della raccolta (Les amours bors de saison e Les respects nuisibles); in secondo luogo, la creazione di una cornice che leghi fra esse e racchiuda in forma definitiva le ormai sette novelle. Ad ognuna di queste categorie - eccettuata la prima, cui è consacrato uno studio a sé stante di Laura Rescia - l'introduzione riserva ampie e penetranti annotazioni critiche. Tenteremo qui fedelmente di esporle, provando inoltre a enuclearne gli spunti essenziali.

«La modification du titre des nouvelles pique la curiosité et mérite qu'on s'y attarde» (p. 13). Nel lasso di tempo che separa le due edizioni, la trasformazione dei titoli delle singole novelle, così come di quello della raccolta, ubbidisce a una precisa logica di slittamento semantico dal concreto all'astratto, dal particolare all'universale: 
«Ce qui intéresse maintenant notre auteur, c'est d'élargir le discours plutôt que de le préciser, de le généraliser plutôt que de le concentrer sur un fait singulier. Il ne caractérise plus chaque nouvelle par la référence concrète aux principaux personnages qui y figurent, il en retient plutôt un trait essentiel qu' il met en relief dans le titre. Au lieu d'un "pauvre doté de générosité”, il évoque "la vertu récompensée"; au lieu d'une "sœur tourmentée", il évoque "la jalousie cruelle"; au lieu d'un "couple de mal mariés", "les mariages mal assortis”, etc. Au-delà du cas particulier de chaque histoire, c'est sa valeur universelle qui est soulignée» (p. 13). Le variazioni nella disposizione delle cinque novelle originarie all'interno dell'opera sono di meno agevole lettura. La proposta della curatrice d' interpretarle come l'esito di una scelta di raggruppamento e alternanza per categorie drammaturgiche pare pienamente convincente: «Le recueil présente une série de nouvelles dont les quatre premières sont tragicomiques des amours contrariés (ou comiques, dans le sens de l'époque), viennent ensuite une nouvelle "tragique", une nouvelle "tragi-comique d'aventure" et une "pastorale". Je me suis permis d'utiliser une terminologie théàtrale pour définir ces nouvelles parce que c'est ce que Sorel lui-même a fait dans sa présentation Aux lecteurs: "vous trouverez dans ce livre une agréable matière d'entretien avec de beaux sujets pour des pièces de Théâtre"» (p. 15). La constatazione di come, raro esempio tra gli autori di rilievo della sua generazione, Sorel non si sia mai cimentato con la scrittura teatrale, non può, malgrado un apparente paradosso, che confermare la felice intuizione di Daniela Dalla Valle. E in effetti del tutto plausibile che Sorel, forte del precedente delle Novelas ejemplares di Cervantes, principale fonte delle Nouvelles choisies, nonché palinsesto di numerose pièces francesi del Seicento, volesse affidare alle proprie narrazioni brevi la missione di colmare, perlomeno in forma indiretta, un'anomala lacuna della sua carriera di scrittore. È bene ricordare, in proposito, come l'itinerario letterario di Sorel sia stato assunto a paradigma delle difficoltà inerenti allo status allora emergente di autore da due fra $\mathrm{i}$ maggiori storici della cultura francese del Seicento, senz' altro i più attenti al nesso fra letteratura e società: Alain Viala (Naissance de l'écrivain. Sociologie de la littérature à l'âge classique, Paris, Éd. de Minuit, 1985, pp. 234-235) e Christian Jouhaud (Les pouvoirs de la littérature. Histoire d'un paradoxe, Paris, Gallimard, 2000, pp. 161-194). Nell'ottica degli studi sulla nascita della figura moderna dello scrittore, il ponte gettato da Sorel verso un genere da lui mai praticato, ma determinante in termini di affermazione nel campo letterario, rivela i limiti impliciti nell'elezione della forma racconto quale unica espressione creativa, e consente altresì di valutare l'intento strategico, per quanto sovente piuttosto ingenuo, di alcune incursioni teoriche oltre i confini delle competenze acquisite, come comprovato, nel caso dell'autore delle Nouvelles, da un suo avventuroso tentativo di partecipare alla Querelle du Cid rivendicando assoluta ignoranza della Poetica di Aristotele: «Je n'ay jamais leu Aristote, et ne sçay point les règles du théâtre mais je règle le mérite des pièces selon le plaisir que j'y reçoy» (Le jugement du Cid, composé par un Bourgeois de Paris, Marguillier de sa Paroisse, citato in A. Gasté, La Querelle du Cid, pièces et pamphlets publiés d'après les originaux, H. Welter, Paris, 1898, p. 231).

La successiva categoria di varianti comprende i già evocati aggiustamenti dell'ispirazione libertina, ed è forse la più significativa sotto il profilo storiografico. Gli interventi di Sorel sul testo del 1623 si applicano a un ventaglio di temi notevolmente articolato. Si passa da un libertinismo erotico che non indietreggia di fronte all'evocazione di pulsioni omosessuali, al motivo, già centrale nell'Histoire comique de Francion, della générosité, forma di merito contrapposta ai privilegi di nascita, o, ancora, agli sfoghi blasfemi di alcuni personaggi contro l'ingiustizia divina (ma sono presenti anche varianti indipendenti dall' ispirazione libertina iniziale, come esemplificato dal più prudente trattamento narrativo che, nel 1645, Sorel riserva al tema del duello, divenuto nel frattempo oggetto di controversie politiche e letterarie). Pur sottolineando gli inconvenienti di questa scelta di autocensura, Daniela Dalla Valle dimostra come, non diversamente da quanto accade nel caso del Francion, gli accorgimenti cautelativi dell'autore non bastino a tacciare d'impoverimento o banalizzazione l'edizione del 1645. La maturazione del testo comporta, certo, una perdita di freschezza semantica e vigore ideologico, ma si accompagna anche a una più rigorosa veste stilistica (come puntualmente rilevato nella preziosa Analyse linguistique di Laura Rescia) e a una meglio definita organizzazione narrativa.

E precisamente di quest'ultimo aspetto che tratta la curatrice commentando l'aggiunta di due novelle al principio e in coda alla raccolta, nonché l'inserzione di passaggi metadiegetici con funzione di cornice. Gli apporti e le modificazioni alla struttura dell'opera configurano una diversa concezione estetica, da un lato, regressivamente prossima ai modelli rinascimentali di ascendenza boccacciana, dall'altro, già volta in direzione di una futura poetica del distanziamento, l'art de l'éloignement, secondo la formula di un fortunato saggio sul classicismo francese (Thomas Pavel, L'art de l'éloignement. Essai sur l'imagination classique, Gallimard, Paris, 1996). E esemplare, al riguardo, il testo inserito alla fine dell'edizione del 1645, Les respects nuisibles. In questa novella narrata alla prima persona da un personaggio della cornice, Sorel evoca, sotto 
il tenue velo di un'ambientazione pastorale, la propria adolescenza trascorsa fra precoci prove poetiche di matrice galante e contatti con il milieu libertino riunito attorno alla carismatica figura di Théophile de Viau. Il tema centrale del racconto, ovvero un'infelice, pudica e ostinata passione adolescenziale, si presta, grazie alla penna ormai esperta dell'autore, all'impiego di una tonalità narrativa in minore, esitante fra l'ironico e il melanconico, in maniera non dissimile, come osserva Daniela Dalla Valle, da quanto avviene nel Page disgracié (1643) di Tris-tan L'Hermite, altro autore formatosi nel pieno della temperie libertina e orientatosi in seguito, non è dato sapere con certezza se per autentico convincimento o motivazioni prudenziali, verso un' espressione neo-stoica di umanesimo cristiano. L'adozione di questo registro distanziato non solo consente all'autore di rivisitare criticamente la stagione del libertinismo flamboyant e delle mode arcadiche in voga all'Hôtel de Rambouillet, ma gli offre anche lo spunto per riflessioni teoriche sul principio della naïveté, nozione che troverà ulteriori sviluppi nella Bibliothèque française (1664) e nella Connaissance des bons livres (1671). Les respects nuisibles si chiude così sulle reazioni dell'uditorio a proposito della maggiore verosimiglianza del racconto di Panfhile rispetto a quelli in precedenza ascoltati: «Panfhile ayant ainsi raconté son histoire, chacun avoua que l'on n'en pouvait entendre une plus naïve; qu'il n'y avait pas des aventures étranges comme il en peut arriver à des personnes qui courent le pays, ni de grandes intrigues comme à des courtisans raffinés, ni des combats et autres entreprises hardies, comme celle des hommes âgés et résolus; mais que c'étaient de vraies affections d'un jeune homme, qui n'a pas encore appris tonte la malice du monde» (p. 412). Memore di una già sperimentata intelligenza critica del testo (si vedano, fra altre pubblicazioni, Le nuove novelle $d i$ Charles Sorel [1645], in La «guirlande» di Cecilia. Studi in onore di Cecilia Rizza, Schena-Nizet, Fasano-Paris, 1996, pp. 385-395; e "Les respects nuisibles: una novella pastorale di Charles Sorel", in Pastorale italiana-Pastorale francese, supplemento a «Franco-Italica», 1996, pp. 159-166), Daniela Dalla Valle si mostra particolarmente attenta a questo carattere di hapax narrativo, mettendone in luce il contributo determinante alla trasformazione del senso generale della raccolta. Ad immagine di quest'ultima novella, l'edizione del 1645 si contraddistingue, in ultima analisi, per un processo relativamente uniforme di "smorzatura", si svolga esso sul piano linguistico, ideologico o narrativo. Le libertà verbali, gli accenti eterodossi e l'esuberanza narrativa di un giovane "io" autoriale che s'impone come unico elemento di connessione fra i diversi racconti lasciano il campo a un'operazione sapiente, sep- pure a tratti un poco insipida, di riordino strutturale, tematico e stilistico. Come suggerito dalla curatrice, sarebbe inopportuno accordare un'univoca preferenza alla prima o alla seconda versione; entrambe presentano irrinunciabili connotati estetici, e una corretta lettura dell'opera non può prescindere da questa sua natura mobile e stratificata. È d'altronde su tale presupposto che si fonda l'utile scelta di corredare il testo del 1645 non solo con un apparato completo delle varianti del 1623, ma anche con numerose note in cui è proposta un'interpretazione dei più significativi fra gli interventi d'autore; «le choix d'un seul recueil par rapport à l'autre», glossa Daniela Dalla Valle, «ne peut être absolu ni définitif: la seconde version, quoiqu'améliorée sur le plan formel, ne peut être justement interprétée si on ne la rapproche pas de la première, en la comparant à celle-ci dans plusieurs occasions, surtout sur le plan idéologique» (p. 24).

Il Sorel che emerge tra le pieghe dell' edizione critica di Daniela Dalla Valle si situa a distanza intermedia fra l'immagine accreditatane dai primi commentatori, Émile Roy e Antoine Adam, propensi a svalutare con indebita fretta la maturazione del giovane libertino, e quella ricostruita da alcuni interpreti recenti, i quali, per reazione alla vulgata, hanno talvolta ecceduto nel tentativo di attribuire all'autore una forte coerenza attraverso gli anni (ci sembra questo il caso dei pure imprescindili contributi di Fausta Garavini, secondo cui Sorel «non cessa di sognare i suoi giovani sogni, lavorando interminabilmente a un'unica opera, frammentandola e suddividendola in libri diversi, che appaiono come singoli capitoli, stesure parziali, brogliacci preparatori», La casa dei giochi. Idee e forme nel Seicento francese, Einaudi, Torino, 1980, p. 142). Ne risulta il ritratto in filigrana di uno scrittore sensibile alle pressioni della sua epoca e pronto al cambiamento, ma non pertanto incline a facili palinodie; uno scrittore, in breve, la cui evoluzione rispecchia il destino di grande parte della corrente libertina, combattuta fra desiderio di fedeltà alle origini e necessità di un ritorno all' ordine.

Grazie a questa edizione critica delle Nowvelles choisies, è ormai posta la prima pietra del monumento tardivamente innalzato a uno fra i massimi narratori del Seicento. Prolungando, e ribaltando al tempo stesso, una metafora già impiegata da Sorel al fine di prevenire gli attacchi dei suoi detrattori («je vous declare que comme l'edifice est fresle et de peu de prix, le fondement n'est pas plus à estimer», La Maison des Jeux, N. de Sercy, Paris, 1642, pp. 403-404), concluderemo col dire che le fondamenta gettate da Daniela Dalla Valle per la costruzione di questo edificio non potrebbero essere più solide.

[FILIPPO D'ANGELO] 